\title{
USO DE UN SOFTWARE ELABORADO MEDIANTE PROGRAMACIÓN VISUAL BASIC PARA EL ANÁLISIS DE ESTRUCTURAS.
}

Por:

Jorge Fernando Márquez Peñaranda', Alexis Quiroga Londoño²

\section{RESUMEN}

Este trabajo presenta el uso de un software desarrollado mediante programación en lenguaje Visual Basic para el análisis de estructuras a través del método de la rigidez. Las estructuras que se pueden calcular usando este software son tipo pórtico plano, pórtico espacial, cercha plana, cercha espacial y placas reticulares. Para la validación del software, se realizó el análisis estructural de un pórtico plano usando el software comercial SAP2000 versión 11 y luego se comparó con el encontrado por el software desarrollado para este trabajo, no encontrándose una diferencia significativa entre los valores calculados, lo cual da confiabilidad al uso del software desarrollado. El uso de este software en investigación y docencia de cursos en ingeniería civil permite cumplir con un doble objetivo, primero que pueda ser usado como herramienta de cálculo y segundo que sirva como punto de partida para enseñanza de algoritmos y generación de códigos que lo modifiquen y mejoren desde el aula.

\section{ABSTRACT}

This work presents the use of a software developed using Visual Basic language for the analysis of structures based on the stiffness method. With this program can be calculated structures like plane frame, spatial frame, plane truss, spatial truss, and reticular plates. To get the software validation, a plane frame was analyzed using software SAP2000 v. 11 and its output values were compared with those obtained from the use of the software developed. In this comparison was not encountered any important difference between values calculated guaranteeing realibility with the use of the developed software. The use of this software in researching projects and courses for civil engineering allows to accomplish two objectives: first, the program can be used like a calculation help and second, It has to be useful to teach algorithms and generation of codes those which can produce changes and improvements into program when it is studied in the classroom.

\section{PALABRAS CLAVE:}

Método de la rigidez, Visual Basic, Estructuras reticulares

\section{KEY WORDS:}

Stiffness method, Visual Basic, Reticular structures

\section{INTRODUCCIÓN}

El avance de la tecnología ha hecho que en el tiempo actual el modo de vivir sea más cómodo pues ha logrado alta economía en insumos, tiempo y dinero, y ha aumentado la eficiencia con la que se hacen todo tipo de tareas al servicio de la sociedad.

El computador es una herramienta que ha contribuido desde su aparición a que las ciencias tengan un ritmo acelerado en su crecimiento. En el campo de la ingeniería ha sido un fuerte aliado para la solución de cálculos que anteriormente se hacían manualmente y consumían enormes cantidades de tiempo conllevando errores frecuentes propios del cansancio. Con el desarrollo del álgebra lineal y la ingeniería de software hoy es posible resolver problemas con precisión y confiabilidad en cuestión de fracciones de segundos que antes requerían de tiempo dispendioso. En la actualidad, la comunidad académica que desarrolla, adapta o usa métodos de análisis estructural coinciden en que tanto los modelos sofisticados, o aquellos menos rigurosos son importantes según su campo de

\footnotetext{
${ }^{1}$ Ingeniero Civil, MSc. Profesor investigador U.F.P.S. Director grupo de investigación Construcciones Civiles Armónicas. jfmarque@ bari.ufps.edu.co

2 Ingeniero Civil U.F.P.S. aql@hotmail.com
} 


\section{USO DE UN SOFTWARE ELABORADO MEDIANTE PROGRAMACIÓN VISUAL BASIC PARA EL ANÁLISIS DE ESTRUCTURAS.}

aplicación. Esto se traduce en una nueva necesidad: Repensar el currículo en la enseñanza del análisis estructural que involucre una fuerte formación teórica y a la vez permita sacar el máximo provecho a las ayudas computacionales no solo aplicando software existente sino mejor aun desarrollando nuevo software o adaptando el existente $[1,2,3]$.

La ciencia se retroalimenta con el desarrollo tecnológico y gracias a éste hoy en día existen potentes programas comerciales para el análisis de estructuras tales como el SAP2000, el RCBuilding, el ETHABS o el SYSMICAD que facilitan las labores de análisis, diseño e investigación propias de la teoría estructural. La Universidad Francisco de Paula Santander (U.F.P.S.) participa activamente en el desarrollo tecnológico y como tal ha diseñado un software de análisis de estructuras por el método de la rigidez usando el lenguaje de programación Visual Basic, que trabaja en un ambiente gráfico, lo que facilita al estudiante o al ingeniero analista realizar ingreso de datos de una estructura para que sea analizada y a su vez sea visible la geometría, los diagramas y resultados numéricos en una interfase gráfica. Un aporte importante del programa es la inclusión de rotación de apoyos que usualmente no se considera de manera implícita en otros programas comerciales.

Por lo anterior en este trabajo, se presentan los aspectos teóricos del método de rigidez, una descripción del software desarrollado y la validación de éste para el análisis estructural de un pórtico plano. Resultados que son comparados con los hallados para este mismo tipo de estructura usando el software comercial SAP2000 versión 11.

\section{ASPECTOS TEÓRICOS}

El método de la rigidez es un procedimiento decantado de la teoría de los elementos finitos en el cual un sistema estructural se estudia por medio de variables asociadas a su equilibrio estático (fuerzas) y a sus características cinemáticas (desplazamientos) [4]. Así es posible plantear un sistema matricial que relacione la rigidez de los elementos (barras) de la estructura con los desplazamientos que ocurren en sus nudos. Usando por ejemplo la energía de deformación [5] o un producto de ésta como el método de pendientedeflexión (slope-deflection) [6] puede obtenerse la matriz de rigidez de una barra para cualquier estructura reticular. La figura 1 muestra los grados de libertad para una barra de sistema estructural tipo pórtico espacial (tres desplazamientos por nudo y tres giros por nudo). Esta totalidad de grados de libertad podría usarse en cualquier tipo de estructura reticular con un adecuado condicionamiento para estructuras menos complejas (por ejemplo en una cercha plana). Sin embargo el aumento del consumo de recursos de software y tiempo hace que en la práctica se planteen y analicen subrutinas que consideren modelos simplificados para los diferentes tipos de estructuras diferentes al pórtico espacial. De cualquier forma los algoritmos para plantear las matrices de rigidez suelen partir de la ecuación (1) [5] y posteriormente condicionar el sistema con la conectividad de barras, con los desplazamientos conocidos y desconocidos y con las posibles transformaciones en los apoyos de la estructura (por ejemplo rotaciones).

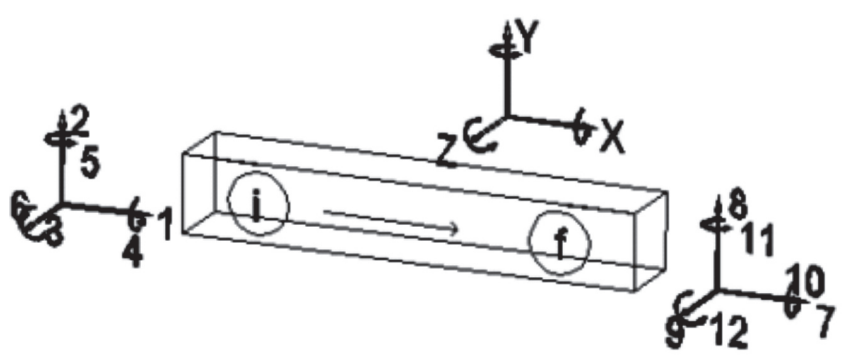

Figura 1. Grados de libertad en un elemento de pórtico tridimensional

$U=\frac{f_{x}{ }^{2} L}{2 A E}+\int_{l} \frac{m_{y}{ }^{2}}{2 E I_{y}} d x+\int_{l} \frac{m_{z}{ }^{2}}{2 E I_{z}} d x+\int_{l} \frac{m_{x}{ }^{2}}{2 G I_{x}} d x+\eta \int_{l} \frac{V_{y}{ }^{2}}{2 G A} d x+\eta \int_{l} \frac{V_{z}{ }^{2}}{2 G A} d x$

En la ecuación (1) $f_{x}, m_{x}, m_{z}, m_{y}, V_{y}, y V_{z}$ son el valor de fuerza axial y las funciones de momento torsor, 


\section{USO DE UN SOFTWARE ELABORADO MEDIANTE PROGRAMACIÓN VISUAL BASIC PARA EL ANÁLISIS DE}

\section{ESTRUCTURAS.}

momento flector alrededor del eje Z, momento flector alrededor del eje $Y$, fuerza cortante en dirección $Y$ y fuerza cortante en dirección $Z$ respectivamente. El valor $\eta$ es un parámetro de ajuste que depende de la forma de la sección y $U$ es la energía de deformación total de un elemento tridimensional elástico y de sección constante [5].

En términos generales el método de rigidez consiste en resolver la ecuación matricial (2) donde $\left\{\bigcup_{0}\right\}$ es el vector de desplazamientos desconocidos, $\left\{\bigcup_{1}\right\}$ es el vector de desplazamientos conocidos o de condiciones cinemáticas, $\left[K_{0}\right],\left[K_{1}\right],\left[K_{2}\right]$ y $\left[K_{3}\right]$ son submatrices de rigidez formadas a partir de la matriz de rigidez ensamblada para toda la estructura de acuerdo con el condicionamiento cinemático, $\left\{\mathrm{F}_{0}\right\}$ es el vector de fuerzas equivalentes de nudo asociadas a los desplazamientos desconocidos y $\left\{\mathrm{F}_{1}\right\}$ es el vector de fuerzas equivalentes de nudo asociadas a los desplazamientos conocidos donde normalmente aparecen las fuerzas de reacción en los apoyos [3] .

$$
\begin{aligned}
\left\{\frac{F_{O}}{F_{1}}\right\}=\left[\begin{array}{c:c}
K_{0} & K_{1} \\
\hdashline K_{2} & K_{3}
\end{array}\right] \times\left\{\frac{U_{O}}{U_{1}}\right\} \\
\therefore\left\{U_{O}\right\}=\left[K_{O}\right]^{1} \times\left(\left\{F_{O}\right\}-\left[K_{1}\right] \times\left\{U_{1}\right\}\right) \\
\left\{F_{1}\right\}=\left[K_{2}\right] \times\left\{U_{O}\right\}+\left[K_{3}\right] \times\left\{U_{1}\right\}
\end{aligned}
$$

El sistema de ecuaciones (2) tiene variables (desplazamientos y fuerzas) referidas a un sistema global de coordenadas que es común para todas las barras y nudos. Sin embargo en una estructura reticular las barras pueden tener diversos vectores dirección y de allí los grados de libertad en cada uno de sus extremos pueden ser referidos a un sistema de ejes particular para cada barra denominado sistema local lo cual resulta más sencillo. Usando una transformación lineal del sistema global sobre el sistema local es posible después hacer operaciones directas sobre las fuerzas y desplazamientos así obtenidos. La transformación mencionada se logra mediante una matriz de rotación [T] que relaciona los desplazamientos y fuerzas globales en cada extremo de barra con sus equivalentes locales:

$$
\left\{U_{L}\right\}=[T] \times\left\{U_{G}\right\} ; \quad\left\{F_{L}\right\}=[T] \times\left\{F_{G}\right\}
$$

siendo $\left\{U_{L}\right\}$ el vector de desplazamientos locales, $\left\{U_{G}\right\}$ el vector de desplazamientos globales, $\left\{F_{L}\right\}$ el vector de fuerzas locales, $\left\{F_{G}\right\}$ el vector de fuerzas globales.
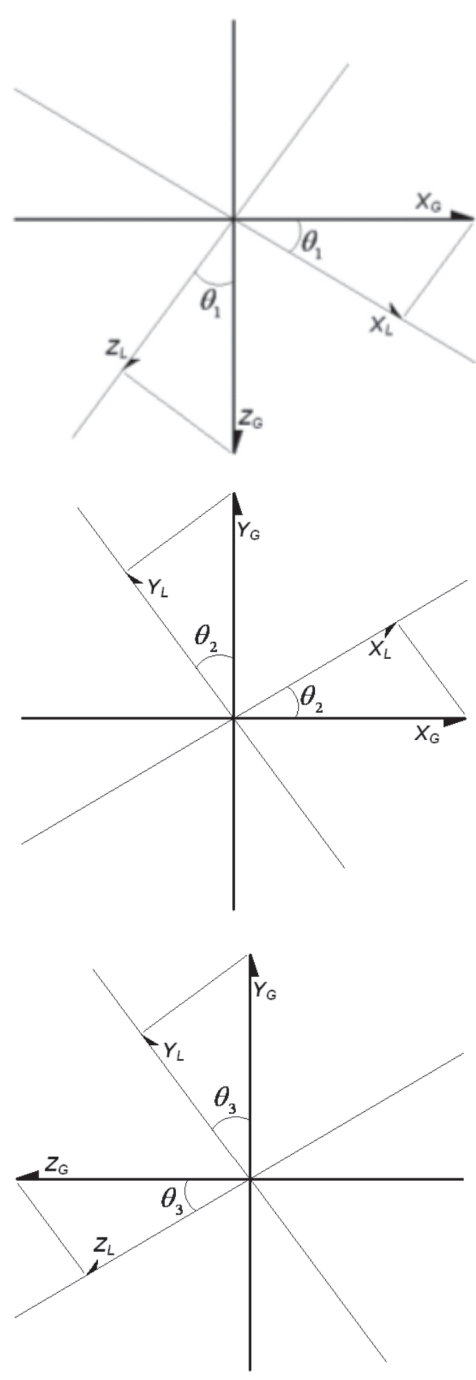

Figura 2. Rotaciones sucesivas sobre elemento de pórtico tridimensional. Adaptada de [6]. 


\section{USO DE UN SOFTWARE ELABORADO MEDIANTE PROGRAMACIÓN VISUAL BASIC PARA EL ANÁLISIS DE}

\section{ESTRUCTURAS.}

El caso más general de este tipo de transformación se asocia a la rotación para una barra de pórtico espacial como se muestra en la figura 2 y en las ecuaciones (4) [6]. Se observa en esa figura que la rotación se hace en tres etapas. Primero se gira el sistema alrededor del eje $Y_{G}$ con un ángulo $\theta_{1}$, luego se induce el giro alrededor del eje $Z_{G}$ con un ángulo $\theta_{2}$ y se termina con un giro alrededor del eje $X_{G}$ con un ángulo $\theta_{3}$. Las proyecciones de los vectores globales (subíndice G) se hacen sobre los vectores locales (subíndice $\mathrm{L}$ ) resultando en cada caso una submatriz de transformación Ri [6]. El sentido de este orden de cada proyección nace de la necesidad práctica de obtener desplazamientos locales y con ellos fuerzas internas locales a partir de unos desplazamientos globales conocidos.

$\begin{array}{ccc}{\left[R_{1}\right]=\left[\begin{array}{ccc}\operatorname{COS} \theta_{1} & -\operatorname{SEN}_{1} & 0 \\ \operatorname{SEN}_{1} & \operatorname{COS} \theta_{1} & 0 \\ 0 & 0 & 1\end{array}\right]} & {\left[R_{2}\right]=\left[\begin{array}{ccc}\operatorname{COS} \theta_{2} & 0 & -\operatorname{SEN} \theta_{2} \\ 0 & 1 & 0 \\ \operatorname{SEN} \theta_{2} & 0 & \operatorname{COS} \theta_{2}\end{array}\right]} \\ {\left[R_{3}\right]=\left[\begin{array}{ccc}1 & 0 & 0 \\ 0 & \operatorname{Cos} \theta_{3} & -\operatorname{SEN}_{3} \\ 0 & \operatorname{SEN}_{3} & \operatorname{COS} \theta_{3}\end{array}\right]} & {[t]=\left[R_{1}\right] \times\left[R_{2}\right] \times\left[R_{3}\right]}\end{array}$

$$
[T]=\left[\begin{array}{cccc}
t & 0 & 0 & 0 \\
0 & t & 0 & 0 \\
0 & 0 & t & 0 \\
0 & 0 & 0 & t
\end{array}\right]_{12 \times 12}
$$

Una de las condiciones cinemáticas especiales suele ser la inclusión de apoyos con rodillos inclinados (figura 3) que crean una dependencia entre desplazamientos ortogonales por lo que es necesario hacer el siguiente ajuste:

$$
\left[K_{r}\right]=\left[R^{G}\right]^{T} \times[K] \times\left[R^{G}\right]
$$

Donde $\left[R^{G}\right]$ es la matriz de rotación global de la estructura considerando una rotación interna de la submatriz que se asocia al apoyo inclinado, $[\mathrm{K}]$ es la matriz de la estructura sin reordenar por condiciones cinemáticas y $[\mathrm{Kr}$ ] es la matriz de la estructura ajustada según la inclinación de sus apoyos.

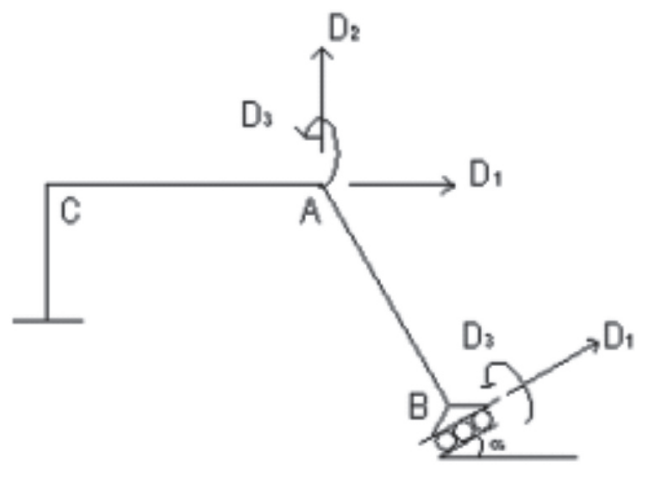

Figura 3. Apoyo con rodillo inclinado

\section{DESCRIPCIÓN Y VALIDACIÓN DEL SOFT- WARE DESARROLLADO}

Una forma eficiente de optimizar los recursos de software consiste en usar operadores propios para diferentes condiciones de borde de los elementos de la estructura. Este software ha sido desarrollado considerando seis operadores según combinaciones de diferentes tipos de apoyos para una barra típica [7]. Sin embargo el usuario puede elegir entre esta opción - aquella que le permita definir las restricciones de borde. Por ejemplo un apoyo articulado en el extremo izquierdo de una barra de pórtico plano puede introducirse también haciendo los desplazamientos horizontal y vertical iguales a cero.

La programación orientada a objetos que usa el Visual Basic exige un código robusto para esta aplicación particular. Además ofrece encapsulamiento que garantiza cierta protección a manera de una caja negra permaneciendo oculta la estructura interna del programa [8]. Esto de alguna manera permite la protección de la propiedad intelectual de la Universidad Francisco de Paula Santander y la habilita para futuras mejoras y actualizaciones del software.

Los dispositivos del programa se clasifican en subrutinas de entrada, subrutinas de proceso y subrutinas de salida. En la tabla 1, se presenta la descripción de 
USO DE UN SOFTWARE ELABORADO MEDIANTE PROGRAMACIÓN VISUAL BASIC PARA EL ANÁLISIS DE ESTRUCTURAS.

los procesos de entrada y salida. Todos estos son muy amigables con el usuario, por ejemplo en la figura 4, se presenta la entrada de datos para secciones de barras y coordenadas de nudos. estructuras probadas incluyeron por lo menos dos de cada tipo: Viga (como caso particular de pórtico plano), Pórtico Plano, Pórtico Tridimensional, Placa Reticular, Cercha Plana y Cercha Tridimensional. El programa

\begin{tabular}{|l|l|}
\hline & \multicolumn{1}{|c|}{ ENTRADAS } \\
\hline NUEVO & Permite ingresar una nueva estructura \\
\hline ABRIR & Permite abrir un archivo de una estructura guardada \\
\hline GUARDAR & Permite salvar la información en cualquier instante \\
\hline $\begin{array}{l}\text { INSERTAR } \\
\text { NUDOS }\end{array}$ & Permite insertar y borrar nudos con sus coordenadas \\
\hline $\begin{array}{l}\text { INSERTA } \\
\text { BARRAS }\end{array}$ & $\begin{array}{l}\text { Permite insertar y borrar barras y sus condiciones de } \\
\text { Conectividad }\end{array}$ \\
\hline MENÚ CARGAS & Permite ingresar y borrar cargas de varios tipos en nudos y barras \\
\hline $\begin{array}{l}\text { MEN } \\
\text { ESTRUCTURA }\end{array}$ & Permite particularizar el tipo de estructura que se desea analizar \\
\hline MENÚ & Permite ingresar y borrar las condiciones de borde \\
\hline $\begin{array}{l}\text { MENÚ } \\
\text { SECCIONES }\end{array}$ & $\begin{array}{l}\text { Permite elegir la sección de cualquier barra introduciendo manualmente } \\
\text { una geometría irregular o regular o seleccionando la sección de una base } \\
\text { de datos }\end{array}$ \\
\hline $\begin{array}{l}\text { MEN } \\
\text { DIAGRAMAS }\end{array}$ & $\begin{array}{l}\text { Permite elegir el tipo de diagrama según el efecto (fuerza axial, momento } \\
\text { torsor, flexión en dos ejes, cortante en dos ejes que se desea observar } \\
\text { para las diferentes barras de la estructura. Además puede solicitarse el } \\
\text { dibujo de la estructura deformada (elástica) }\end{array}$ \\
\hline $\begin{array}{l}\text { TRASLADAR } \\
\text { IMAGEN }\end{array}$ & Permite mover la imagen hacia los lados o hacia arriba y abajo \\
\hline $\begin{array}{l}\text { ROTAR } \\
\text { IMAGEN }\end{array}$ & Permite girar la imagen para observarla en otra perspectiva \\
\hline $\begin{array}{l}\text { AUMENTAR } \\
\text { IMAGEN }\end{array}$ & Permite aumentar el tamaño de la imagen \\
\hline $\begin{array}{l}\text { REDUCIR } \\
\text { IMAGEN }\end{array}$ & Permite reducir el tamaño de la imagen \\
\hline VISTA INICIAL & Retorna la imagen a su presentación original \\
\hline IMPRIMIR & Permite imprimir imágenes o texto de resultados \\
\hline
\end{tabular}

Tabla 1. Descripción de los procesos de entrada y salida del software desarrollado.

La validación del programa se hizo usando diferentes condiciones de carga y de borde. Además se intentaron posibles ingresos de datos erróneos o ausencia de éstos así como corridas de programa parciales. Las no presentó errores de ejecución por contradicciones internas y la precisión de sus resultados es comparable a la obtenida con otros programas comerciales tales como el SAP2000 versión 11. 
USO DE UN SOFTWARE ELABORADO MEDIANTE PROGRAMACIÓN VISUAL BASIC PARA EL ANÁLISIS DE ESTRUCTURAS.

\section{BASE DE DATOS}

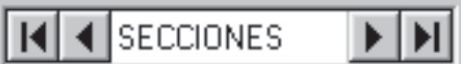

\begin{tabular}{|l}
\hline ID \\
\hline SECCION \\
\hline$x[\mathrm{~cm} 4]$ \\
\hline$y(\mathrm{~cm} 4)$ \\
\hline$z(\mathrm{~cm} 4)$ \\
\hline$(\mathrm{cm} 2)$
\end{tabular}
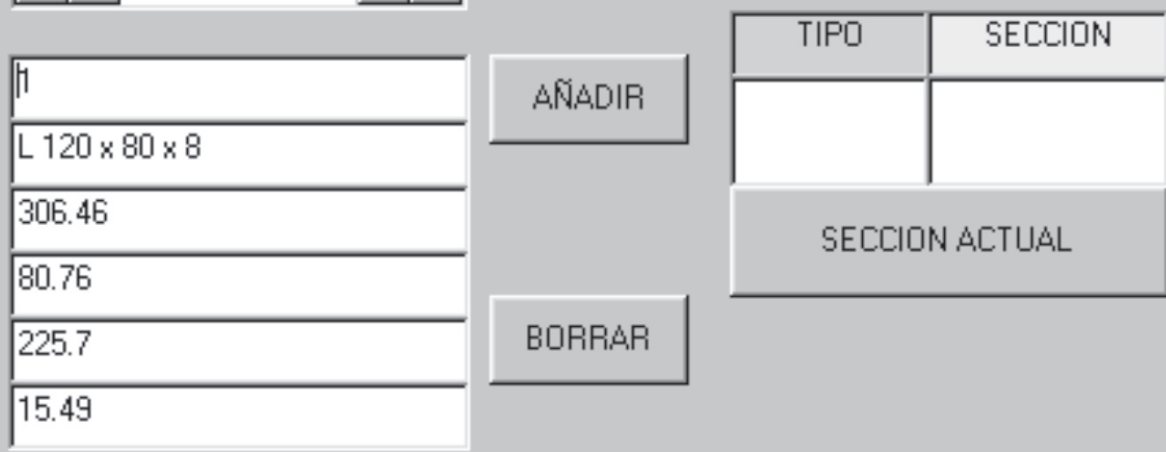

\section{SECCION ACTUAL}

BUSCAR
C Buscar por el campo ID
C Buscar por el campo SECCION

\section{BUSCAR}

\begin{tabular}{|c|c|c|}
\hline & \begin{tabular}{l|l} 
ID & SECCIONES \\
\end{tabular} & $\mathrm{Ix}[\mathrm{cm} 4] \mid \mathrm{ly}[\mathrm{c}-$ \\
\hline$\nabla$ & 1 L $120 \times 80 \times 8$ & 306.4680 .7 \\
\hline & $2 \mathrm{~L} 120 \times 80 \times 10$ & 373.6198 .1 \\
\hline & $3 \mathrm{~L} 120 \times 80 \times 12$ & \begin{tabular}{l|l}
437.1 & 114 \\
\end{tabular} \\
\hline & $4 \mathrm{~L} 130 \times 65 \times 8$ & 307.2744 .7 \\
\hline & $5 \mathrm{~L} 130 \times 65 \times 10$ & 374.754 .2 \\
\hline & 6 L $150 \times 90 \times 10$ & \begin{tabular}{ll|l}
679.2 & 146
\end{tabular} \\
\hline & $7 \mid 15 \cap$. $90 \times 11$ & 739 A1159 \\
\hline 1 & & 1 \\
\hline
\end{tabular}

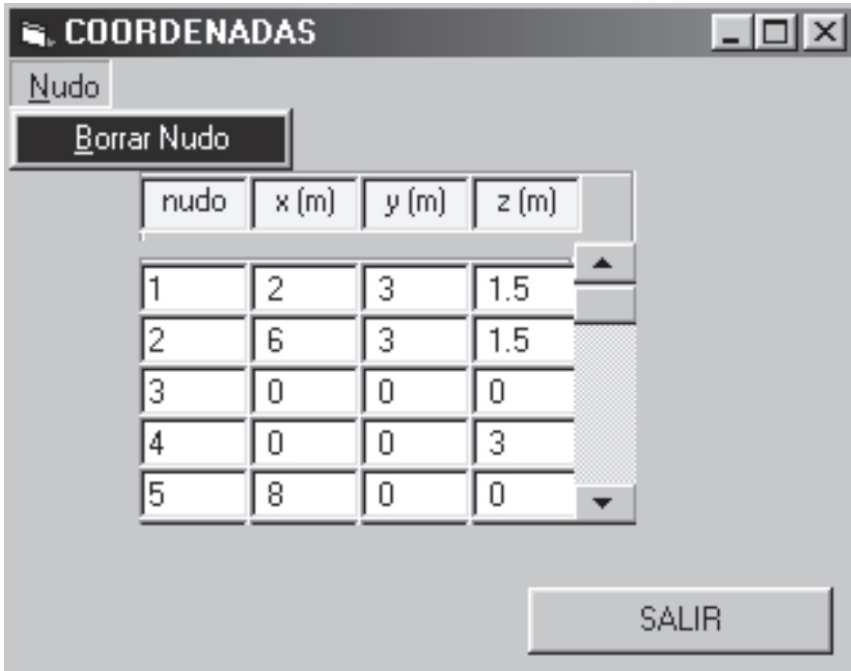

Figura 4. Ejemplo de entrada de datos para secciones de barras y coordenadas de nudos. 


\begin{tabular}{|c|c|c|c|c|}
\hline \multicolumn{2}{|c|}{ DESPLAZAMIENTOS } & PROGRAMA & SAP2000 & ERROR \\
\hline \multirow{3}{*}{ NUDO 1} & Ux & 0 & 0 & $0 \%$ \\
\hline & Uy & $-0,0200$ & $-0,0200$ & $0 \%$ \\
\hline & Giro & $-0,0070$ & $-0,0071$ & $-1,0 \%$ \\
\hline \multirow{3}{*}{ NUDO 2} & Ux & 0 & 0 & $0 \%$ \\
\hline & Uy & 0 & 0 & $0 \%$ \\
\hline & Giro & 0 & 0 & $0 \%$ \\
\hline \multirow[t]{3}{*}{ NUDO 3} & Ux & 0,0216 & 0,0218 & $-1,0 \%$ \\
\hline & Uy & 0,0164 & 0,0164 & $0,1 \%$ \\
\hline & Giro & $-0,0108$ & $-0,0108$ & $-0,2 \%$ \\
\hline \multirow[t]{3}{*}{ NUDO 4} & Ux & 0,0270 & 0,0273 & $-1,2 \%$ \\
\hline & Uy & $-0,0002$ & $-0,0002$ & $-0,1 \%$ \\
\hline & Giro & $-0,0117$ & $-0,0118$ & $-0,2 \%$ \\
\hline \multirow[t]{3}{*}{ NUDO 5} & Ux & 0,0339 & 0,0344 & $-1,5 \%$ \\
\hline & Uy & $-0,0203$ & $-0,0203$ & $0 \%$ \\
\hline & Giro & $-0,0029$ & $-0,0029$ & $-1,5 \%$ \\
\hline \multicolumn{2}{|c|}{ REACCIONES } & PROGRAMA & SAP2000 & ERROR \\
\hline \multirow{3}{*}{ NUDO 1} & $F x$ & $-44,78$ & $-44,83$ & $-0,1 \%$ \\
\hline & Fy & 279,27 & 279,16 & $0,0 \%$ \\
\hline & $\mathrm{M}$ & 0,00 & 0,00 & $0 \%$ \\
\hline \multirow[t]{3}{*}{ NUDO 2} & $\mathrm{Fx}$ & $-155,22$ & $-155,17$ & $0,0 \%$ \\
\hline & Fy & 170,75 & 170,84 & $-0,1 \%$ \\
\hline & $M$ & 382,10 & 382,36 & $-0,1 \%$ \\
\hline \multicolumn{2}{|c|}{ FUERZAS INTERNAS } & PROGRAMA & SAP2000 & ERROR \\
\hline \multirow{6}{*}{ BARRA 1} & $A i$ & 170,75 & 170,84 & $-0,1 \%$ \\
\hline & Vi & 155,22 & 155,17 & $0,0 \%$ \\
\hline & Mi & 382,10 & 382,36 & $-0,1 \%$ \\
\hline & $A f$ & $-170,75$ & $-170,84$ & $-0,1 \%$ \\
\hline & $V f$ & $-155,22$ & $-155,17$ & $0,0 \%$ \\
\hline & $M f$ & 83,57 & 83,14 & $0,5 \%$ \\
\hline \multirow{6}{*}{ BARRA 2} & $A f$ & $-279,27$ & $-279,16$ & $0,0 \%$ \\
\hline & $V f$ & $-44,78$ & $-44,83$ & $-0,1 \%$ \\
\hline & $M f$ & 0,00 & 0,00 & $0 \%$ \\
\hline & $A i$ & 279,27 & 279,16 & $0,0 \%$ \\
\hline & Vi & 44,78 & 44,83 & $-0,1 \%$ \\
\hline & Mi & 268,70 & 269,00 & $-0,1 \%$ \\
\hline \multirow{6}{*}{ BARRA 3} & $A i$ & 0,00 & 0,00 & $0,0 \%$ \\
\hline & Vi & 0,00 & 0,00 & $0,0 \%$ \\
\hline & Mi & 0,00 & 0,00 & $0,0 \%$ \\
\hline & Af & 28,46 & 28,46 & $0,0 \%$ \\
\hline & $V f$ & 85,38 & 85,38 & $0,0 \%$ \\
\hline & $M f$ & $-67,45$ & $-67,50$ & $-0,1 \%$ \\
\hline \multirow{6}{*}{ BARRA 4} & $A i$ & $-121,70$ & $-121,64$ & $0,1 \%$ \\
\hline & Vi & 125,70 & 125,76 & $0,0 \%$ \\
\hline & Mi & $-15,82$ & $-15,64$ & $1,1 \%$ \\
\hline & Af & 235,58 & 235,48 & $0,0 \%$ \\
\hline & $V f$ & 215,84 & 215,77 & $0,0 \%$ \\
\hline & $M f$ & $-268,70$ & $-269,00$ & $-0,1 \%$ \\
\hline
\end{tabular}

Los resultados mostrados en la tabla 2 corresponden al análisis estructural del pórtico plano mostrado en la figura 5 y fueron obtenidos usando el programa desarrollado por la U.F.P.S. y el software comercial SAP2000 versión 11 . Los valores siguen la convención de ejes positivos $X, Y$ hacia la derecha y hacia arriba respectivamente. Los momentos o giros son positivos en sentido antihorario. La estructura mostrada en la figura citada es de concreto con un $\mathrm{E}=17872 \mathrm{MPa}$ y las barras 1,3 y 4 son prismáticas con sección de $40 \mathrm{~cm} X 40 \mathrm{~cm}$ mientras que la barra 2 tiene $60 \mathrm{~cm} \times 60 \mathrm{~cm}$ de sección. Se ha considerado además de las cargas mostradas $(60 \mathrm{KN} / \mathrm{m}$ en barras 3 y 4 y $200 \mathrm{KN}$ en nudo 5) un desplazamiento de $2 \mathrm{~cm}$ (asentamiento) del apoyo 1 hacia abajo.

Para calcular el error en la tabla 2 se usaron los resultados del programa SAP2000 versión 11 como valores correctos y el porcentaje mostrado corresponde a la diferencia con los calculados por el software desarrollado. En general se observa una buena precisión del programa desarrollado, pues la mayor diferencia no supera 1,5\% y la gran mayoría de errores

Tabla 2. Comparación de resultados obtenidos mediante el programa desarrollado para este trabajo y los calculados con SAP2000v. 11 para la estructura mostrada en la figura 5. son muy próximos a cero. La mayor discrepancia se presenta en los giros pero al final los desplazamientos lineales, las reacciones y fuerzas internas que son de especial interés para diseño muestran una excelente precisión. 


\section{USO DE UN SOFTWARE ELABORADO MEDIANTE PROGRAMACIÓN VISUAL BASIC PARA EL ANÁLISIS DE}

ESTRUCTURAS.

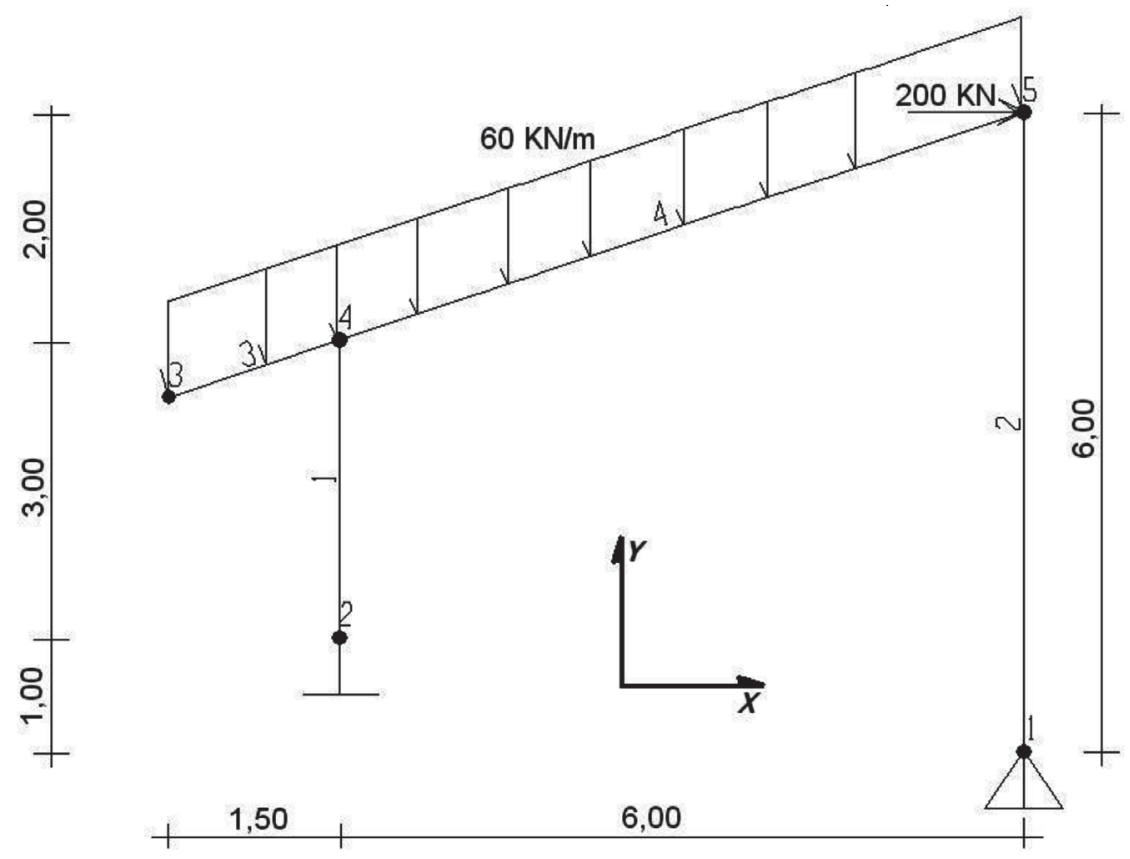

\section{CONCLUSIONES}

El software creado constituye un aporte al desarrollo tecnológico necesario para la investigación de modelos estructurales reticulares y representa una herramienta de avance para la docencia en lo que concierne a enseñanza del análisis estructural.

Como puede observarse en la tabla 2, la precisión de los resultados del programa desarrollado para este trabajo es buena, pues la diferencia media encontrada con respecto al programa SAP2000 versión 11

Figura 5. Estructura usada para cotejar la precisión del programa diseñado con el SAP2000v. 11 y cuyos resultados se muestran en la tabla 2 .

\section{SUGERENCIAS Y RECOMENDACIONES}

Este programa podrá ser usado inicialmente en los cursos de análisis estructural de la Universidad Francisco de Paula Santander para lo cual es conveniente crear un vínculo en la página WEB de la misma donde los estudiantes puedan hacer uso del mismo. También constituye una herramienta para servicios de consultoría, investigación y extensión a la comunidad y el sector productivo. Además sirve como importante herramienta de enseñanza de algoritmos de solución de modelos estructurales en cursos de análisis estructural pues puede modificarse el código del programa como ejercicio de aula para observar los efectos de una sana lógica de programación cuando se usa el método de la rigidez.

La siguiente fase del programa incluirá la introducción de subrutinas para diseño y la creación de posibilidades de importación de archivos de AUTOCAD. oscila alrededor de 0,14\%.

El programa desarrollado permite ingresar, cambiar o borrar datos sin conflicto y sirve para analizar pórticos tridimensionales, pórticos planos, cerchas espaciales, cerchas planas y placas reticulares. Además es posible manejar cualquier clase de apoyo incluso aquellos que se comportan como rodillos inclinados, admite condiciones cinemáticas diversas (desplazamientos conocidos), analiza barras con su sección girada a lo largo de sus ejes longitudinales en las estructuras espaciales, presenta los resultados gráficamente y en hojas de texto y muestra los diagramas de efectos diversos entre otras características que evidencian su versatilidad.

Una muy importante aplicación a la investigación en el aula es constituida por la propiedad intelectual de la U.F.P.S. del programa lo que permite que sus estudiantes y profesores accedan al código del programa y ensayen o propongan modificaciones y mejoras sobre el mismo. Esto garantiza versatilidad en la enseñanza de algoritmos y programación específica para análisis estructural en los cursos de ingeniería civil de la U.F.P.S. 
USO DE UN SOFTWARE ELABORADO MEDIANTE PROGRAMACIÓN VISUAL BASIC PARA EL ANÁLISIS DE

ESTRUCTURAS.

\section{REFERENCIAS BIBLIOGRÁFICAS}

[1] Kitipornchai, S. (2007). Seminar-Instant Structural Analysis: A Tool for Structural Engineering Students and Engineerings. University of Sidney.

[2] Slattery, K. (2004). Computarized Structural Modeling. Journal of American Concrete Institute. Concrete International. Vol 26, No 12. 28-31. United States.

[3] Furlong, R. (2005). Theory of Elastic AnalysisIllusion and Superstition. Journal of American Concrete Institute. Special Publication. Vol 225. 112. United States.

[4] Ibañez de Navarra, E. (1992). Cálculo de estructuras por el método de elementos finitos. 126. Centro Internacional de Métodos Numéricos. España.

[5] Rochel, R. (2000). Análisis Matricial de Estructuras. 2.1-2.17. EAFIT. Colombia.

[6] Salcedo, J. (1987). Análisis Avanzado de Estructuras. 79-115. UIS. Colombia.

[7] Ghali, A. y Neville, A. (1997). Structural analysis A unified classical and matrix approach. E\&FN Spon: London \& New York.

[8] Ceballos, F. (2000). Curso de programación de Visual Basic 6. 14-17. Alfaomega. España.

Fecha de recibido: Junio 12 de 2006

Fecha de aceptación: Noviembre 28 de 2007 